\title{
Scattering Problem for the Supercritical Nonlinear Schrödinger Equation in 1d
}

\author{
By \\ Nakao Hayashi and Pavel I. NaumKin \\ (Osaka University, Japan and UNAM Campus Morelia, Mexico)
}

\begin{abstract}
We consider the one dimensional nonlinear Schrödinger equation $i u_{t}+u_{x x} / 2=f(u), \quad x \in \boldsymbol{R}, t>0, u(0, x)=u_{0}(x), \quad x \in \boldsymbol{R}$, with a super critical nonlinearity $f(u)=\sum_{j \neq 0} f_{j}(u)$, and $f_{j}(u)$ are such that $f_{j}(u)=\lambda_{j}|u|^{\sigma_{j}-j} u^{j}$, where $\lambda_{j} \in \boldsymbol{C}$, $\sigma_{j}>3$. We prove the existence of the scattering operator in the weighted Sobolev spaces.

Key Words and Phrases. Scattering operator, Asymptotic behavior in time, Nonlinear Schrödinger, Power nonlinearity.

2010 Mathematics Subject Classification Numbers. 35Q55, 35B40.
\end{abstract}

\section{Introduction}

We consider the one dimensional nonlinear Schrödinger equation

$$
\left\{\begin{array}{l}
i u_{t}+\frac{1}{2} u_{x x}=f(u), \quad x \in \boldsymbol{R}, t>T, \\
u(T, x)=u_{0}(x), \quad x \in \boldsymbol{R},
\end{array}\right.
$$

with a super critical nonlinearity $f(u)=\sum_{j \neq 0} f_{j}(u)$, and $f_{j}(u)$ are such that

$$
f_{j}(u)=\lambda_{j}|u|^{\sigma_{j}-j} u^{j},
$$

where $\lambda_{j} \in \boldsymbol{C}, \sigma=\min \sigma_{j}>3$ (really, we use the following properties $f_{j} \in \boldsymbol{C}^{2}$ and $f_{j}\left(r e^{i \theta} u\right)=r^{\sigma_{j}} e^{i j \theta} f_{j}(u)$ for all $\left.r>0, \theta \in \boldsymbol{R}\right)$. Here and below by $\sum_{j \neq 0}$ we mean a finite sum over $j \neq 0$.

When $j=1$, global existence, asymptotic behavior, blow up of solutions for (1) were well studied in general space dimension by many authors (see, a text book [2]) due to the gauge invariant property of the nonlinearity. On the other hand, in the case of $j \neq 1$, equation (1) was not studied well. Global in time existence of small solutions for (1) including $j=0$ in general space dimension $n$ was shown in [18] for $\sigma>\sigma_{s}(n)$, where $\sigma_{s}(n)=\left(n+2+\sqrt{4+12 n+n^{2}}\right) /$ $(2 n)$ is known as the Strauss exponent [17]. The optimal time decay estimate

$$
\|u(t)\|_{\mathbf{L}^{\sigma+1}} \leq C|t|^{-(n / 2)(1-2 /(\sigma+1))}
$$


for small solutions was also obtained in [18] by the method of the $\mathbf{L}^{\sigma+1}-\mathbf{L}^{(\sigma+1) / \sigma}$ - time decay estimate for the free Schrödinger evolution group. From the previous works for the gauge invariant nonlinearity, it is known that the critical exponent is $1+2 / n$ which is called the short range exponent for the scattering problem ([7], [2]). Therefore it is conjectured that $1+2 / n$ is the critical case for (1). Indeed the asymptotic profile of solutions for (1) with the nonlinearity $u^{3}$ in one space dimension is different from that of linear problem. This result was shown in [9]. As far as we know, global existence and scattering of solutions were not obtained for case $1+2 / n<\sigma \leq$ $\sigma_{s}(n)$. Our purpose in the present paper is to fill this gap when $n=1$. Our analysis in the present paper requires some regularity condition on the nonlinearity and does not work well for the nonlinearity of the form $f_{0}(u)=\lambda_{0}|u|^{\sigma}$. Hence the problem is still open for higher space dimensions and the case of the nonlinearity $|u|^{\sigma}$ with $\sigma \leq \sigma_{s}(n)$. On the other hand, the nonlinear Klein-Gordon equation which is considered as the relativistic version of (1) including $j=0$ was studied in [5], [8] and the similar problem was solved for $1 \leq n \leq 3$.

In the middle of 80 's, the vector field and the normal form methods were introduced by [13] and [15] to study the large time asymptotics of solutions to quadratic nonlinear Klein-Gordon equations in three space dimensions. Now these methods are very important tools in the field of nonlinear dispersive equations. The algebraic normal form method by [14], [19] was used for the study of the quadratic nonlinear Klein-Gordon equations in two space dimensions. Also the method of [15] was applied to quadratic nonlinear Schrödinger equations in two space dimensions for the nonlinearity $\bar{u}^{2}$, see [3]. These methods do work well for the quadratic or cubic cases but do not work well for the physically important case $|u| u$. Our method stated below is more general than these methods since it can be used for scattering problem with another types of fractional nonlinearities. Our method depends on the equation for the new dependent variable $v(t)=\mathscr{F} M \mathscr{U}(-t) u(t)$ which is written as (4) below. This equation is useful to clarify the difference between the oscillating factor of linear solutions and that of nonlinear terms denoted by $E$. From this equation we find that the derivative of the oscillating factor gains a time growth but integrating it by parts yields a better time decay. Our weighted homogeneous Sobolev space (which is not necessarily $\mathbf{L}^{2}$ ) is chosen carefully on the balance of the time growth by the derivative and time decay via the integration by parts. As a by-product, we find that our function space is close to the invariant space in the short range exponent. This is the main difference between our previous work [9] and the present paper.

If we restrict our attention to the life span of local solutions, we have an application to a system of Schrödinger equation such that 


$$
\begin{aligned}
& i \partial_{t} v_{1}+\frac{1}{2 m_{1}} \partial_{x}^{2} v_{1}=\lambda \overline{v_{1}} v_{2}, \\
& i \partial_{t} v_{2}+\frac{1}{2 m_{2}} \partial_{x}^{2} v_{2}=\mu v_{1}^{2},
\end{aligned}
$$

where $\lambda, \mu \in C \backslash\{0\}$, the mass of particles $m_{1}, m_{2}$ are positive and satisfy a non resonance mass condition

$$
2 m_{1} \neq m_{2}, \quad m_{1} \neq m_{2} .
$$

This system in higher space dimensions was studied in [10], [11] (see also [4]) for the resonance case $2 m_{1}=m_{2}$, which is easier since the operator $J_{m_{j}}=$ $x+i t \partial_{x} / m_{j}$ works well.

To state our result, we introduce the usual Lebesgue space $\mathbf{L}^{p}$ with the norm $\|\phi\|_{\mathbf{L}^{p}}=\left(\int_{\boldsymbol{R}}|\phi(x)|^{p} d x\right)^{1 / p}$ if $1 \leq p<\infty$ and $\|\phi\|_{\mathbf{L}^{\infty}}=\operatorname{ess} \cdot \sup \{|\phi(x)|$; $x \in \boldsymbol{R}\}$. The weighted Sobolev space is defined by

$$
\mathbf{H}_{p}^{m, k}=\left\{\phi \in \mathbf{L}^{2}:\left\|\langle x\rangle^{k}\left\langle i \partial_{x}\right\rangle^{m} \phi\right\|_{\mathbf{L}^{p}}<\infty\right\},
$$

where $m, k \in \boldsymbol{R}^{+}, \quad 1 \leq p \leq \infty, \quad\langle x\rangle=\sqrt{1+|x|^{2}}$. For simplicity we write $\mathbf{H}^{m, s}=\mathbf{H}_{2}^{m, s}, \mathbf{H}^{m}=\mathbf{H}^{m, 0}$. Homogeneous Sobolev space $\dot{\mathbf{H}}^{\alpha, \beta}$ is defined by

$$
\dot{\mathbf{H}}^{\alpha, \beta}=\left\{\phi \in \mathbf{S}^{\prime} ;\|\phi\|_{\dot{\mathbf{H}}^{\alpha, \beta}}=\left\||x|^{\beta}\left(-\partial_{x}^{2}\right)^{\alpha / 2} \phi\right\|_{\mathbf{L}^{2}}<\infty\right\} .
$$

We introduce a closed ball $(\mathbf{X})_{\leq \rho}=\left\{\phi \in \mathbf{X} ;\|\phi\|_{\mathbf{X}} \leq \rho\right\}$. Let $\mathbf{C}(\mathbf{I} ; \mathbf{X})$ be the space of continuous functions from an interval $\mathbf{I}$ to a Banach space $\mathbf{X}$. Different positive constants might be denoted by the same letter $C$.

We consider the Cauchy problem with initial data at $T=1$. Denote by $\mathscr{U}(t)=e^{i t \hat{t}_{x}^{2} / 2}$ the free Schrödinger evolution group.

Theorem 1.1. Assume that $\sigma>3$ and the initial data $u_{0}$ are such that $\mathscr{U}(-1) u_{0} \in \dot{\mathbf{H}}^{0, \gamma} \cap \dot{\mathbf{H}}^{0, \beta}, 0 \leq \gamma<1 / 2<\alpha<1$. Then there exists $\varepsilon_{0}>0$ such that (1) has a unique global solution

$$
\mathscr{U}(-t) u \in \mathbf{C}\left([1, \infty) ; \dot{\mathbf{H}}^{0, \gamma} \cap \dot{\mathbf{H}}^{0, \alpha}\right)
$$

satisfying the estimate

$$
\|\mathscr{U}(-t) u(t)\|_{\dot{\mathbf{H}}^{0, \gamma} \cap \dot{\mathbf{H}}^{0, \alpha}} \leq C t^{\max \{0, \alpha+5 / 2-\sigma\} / 2}
$$

for all $t>1$, and for any $u_{0}$ such that $\left\|\mathscr{U}(-1) u_{0}\right\|_{\dot{\mathbf{H}}^{0, \gamma} \cap \dot{\mathbf{H}}^{0, \beta}}=\varepsilon \leq \varepsilon_{0}$. Moreover for any global solution $u$, there exists a unique scattering state $u_{+} \in \dot{\mathbf{H}}^{0, \gamma} \cap \dot{\mathbf{H}}^{0, \beta}$, with $0 \leq \gamma<1 / 2<\beta<\sigma-5 / 2$, such that

$$
\left\|\mathscr{U}(-t) u(t)-u_{+}\right\|_{\dot{\mathbf{H}}^{0, \gamma} \cap \dot{\mathbf{H}}^{0, \beta}} \rightarrow 0
$$

as $t \rightarrow \infty$. 
Remark 1.1. If we take $\alpha$ satisfying $1 / 2<\alpha<\sigma-5 / 2$, then $\beta=\alpha$ is permitted in Theorem 1.1. Hence there exists a scattering state in $\dot{\mathbf{H}}^{0, \gamma} \cap \dot{\mathbf{H}}^{0, \alpha}$. In the case of $1 / 2<\alpha<\sigma-5 / 2$, estimate (2) gives us $\|\mathscr{U}(-t) u(t)\|_{\dot{\mathbf{H}}^{0, \gamma} \cap \dot{\mathbf{H}}^{0, \alpha}} \leq C$ from which the sharp time decay estimate $\|u(t)\|_{\mathbf{L}^{\infty}} \leq C t^{-1 / 2}$ follows.

Remark 1.2. Define $u_{\lambda}(t)=\lambda^{2 /(\sigma-1)} u\left(\lambda^{2} t, \lambda x\right)$. Note that $u_{\lambda}(t)$ satisfies (1) with the initial data $u_{0 \lambda}(x)=\lambda^{(\sigma-1) / 2} u_{0}(\lambda x)$. We have

$$
\left\|u_{0 \lambda}\right\|_{\dot{\mathbf{H}}^{0,(\sigma-2) / 2}}=\left\|u_{0}\right\|_{\dot{\mathbf{H}}^{0,(\sigma-2) / 2}}
$$

which implies that $\dot{\mathbf{H}}^{0,(\sigma-2) / 2}$ is the so-called invariant space for problem (1). When $\sigma>3$ is close to 3 , then the function space $\dot{\mathbf{H}}^{0, \gamma} \cap \dot{\mathbf{H}}^{0, \alpha}, \gamma<1 / 2<\alpha<$ $\sigma-5 / 2$, in Theorem 1.1 is close to the invariant space.

Our second result concerns the Cauchy problem with initial data at $T=0$.

Theorem 1.2. Assume that $\sigma>3$, the initial data $u_{0}=u(0, x) \in \mathbf{H}^{\delta} \cap \mathbf{H}^{0, \alpha}$, $1 / 2<\alpha<\delta<1$. Then there exists $\varepsilon_{0}>0$ such that (1) has a unique global solution

$$
\mathscr{U}(-t) u \in \mathbf{C}\left([0, \infty) ; \mathbf{H}^{\delta} \cap \mathbf{H}^{0, \alpha}\right)
$$

satisfying the estimate

$$
\|\mathscr{U}(-t) u(t)\|_{\mathbf{H}^{\delta} \cap \mathbf{H}^{0, \alpha}} \leq C\langle t\rangle^{\max \{0, \alpha+5 / 2-\sigma\} / 2}
$$

for all $t>0$, for any $u_{0}$ such that $\left\|u_{0}\right\|_{\mathbf{H}^{\delta} \cap \mathbf{H}^{0, \alpha}}=\varepsilon \leq \varepsilon_{0}$. Moreover for any global solution $u$, there exists a unique scattering state $u_{+} \in \mathbf{H}^{\delta} \cap \mathbf{H}^{0, \beta}$, with $1 / 2<\beta<$ $\sigma-5 / 2$, such that

$$
\left\|\mathscr{U}(-t) u(t)-u_{+}\right\|_{\mathbf{H}^{\delta} \cap \mathbf{H}^{0, \beta}} \rightarrow 0
$$

as $t \rightarrow \infty$.

Remark 1.3. By Theorem 1.2, the inverse wave operator

$$
W_{+}^{-1}: u_{0} \in\left(\mathbf{H}^{\delta} \cap \mathbf{H}^{0, \alpha}\right)_{\leq \varepsilon} \mapsto u_{+} \in\left(\mathbf{H}^{\delta} \cap \mathbf{H}^{0, \alpha}\right)_{\leq \sqrt{\varepsilon}}
$$

is defined. The proofs of Theorems 1.1 and 1.2 work for the final value problem. Therefore we find that there exists the wave operator

$$
W_{-}: u_{-} \in\left(\mathbf{H}^{\delta} \cap \mathbf{H}^{0, \alpha}\right)_{\leq \rho} \mapsto u_{0} \in\left(\mathbf{H}^{\delta} \cap \mathbf{H}^{0, \alpha}\right)_{\leq \sqrt{\rho}} .
$$

We take $\varepsilon=\sqrt{\rho}$, then the scattering operator

$$
S_{-,+}=W_{+}^{-1} W_{-}: u_{-} \in\left(\mathbf{H}^{\delta} \cap \mathbf{H}^{0, \alpha}\right)_{\leq \rho} \mapsto u_{+} \in\left(\mathbf{H}^{\delta} \cap \mathbf{H}^{0, \alpha}\right)_{\leq \rho^{1 / 4}}
$$

is defined. 
The factorization formula from paper [12] for the free Schrödinger evolution group is presented by

$$
\mathscr{U}(t)=e^{i t \delta_{x}^{2} / 2}=M(t) \mathscr{D}(t) \mathscr{V}(t) \mathscr{F},
$$

where $M(t, x)=e^{i|x|^{2} /(2 t)}$, the dilation operator $(\mathscr{D}(t) \phi)(x)=(i t)^{-1 / 2} \phi(x / t)$, and the operator $\mathscr{V}(t)=\mathscr{F} M(t) \mathscr{F}^{-1}$. Here $\mathscr{F} \phi=(2 \pi)^{-1 / 2} \int_{R} e^{-i x \xi} \phi(x) d x$ denotes the Fourier transform of the function $\phi$, and $\mathscr{F}^{-1}$ is the inverse Fourier transformation defined by $\mathscr{F}^{-1} \phi=(2 \pi)^{-1 / 2} \int_{\boldsymbol{R}} e^{i x \xi} \phi(\xi) d \xi$. Formula (3) is useful for studying the large time asymptotic behavior of solutions of the nonlinear Schrödinger equations. We also have

$$
\mathscr{F} \mathscr{U}(-t)=i \mathscr{V}(-t) \bar{E}(t) \mathscr{D}\left(\frac{1}{t}\right)
$$

where $E(t, \xi)=e^{i t|\xi|^{2} / 2}$. Here we have used the commutation identity $\mathscr{D}(1 / t) M(t)=E(t) \mathscr{D}(1 / t)$. Using the new dependent variables $\varphi(t)=$ $\mathscr{F} \mathscr{U}(-t) u(t)$ and

$$
v(t)=\mathscr{F} M \mathscr{U}(-t) u(t)=\mathscr{V}(t) \varphi(t)=i \bar{E}(t) \mathscr{D}\left(\frac{1}{t}\right) u(t),
$$

we represent the solution as

$$
u(t)=\mathscr{U}(t) \mathscr{F}^{-1} \varphi(t)=M(t) \mathscr{D}(t) v(t) .
$$

In the same way as in paper [12] we multiply both sides of $(1)$ by $\mathscr{F} M \mathscr{U}(-t)$ to get

$$
\mathscr{L} v=\sum_{j \neq 0} i^{(1-j) / 2} t^{\left(1-\sigma_{j}\right) / 2} E^{j-1} f_{j}(v),
$$

where $\mathscr{L}=i \partial_{t}+\partial_{\xi}^{2} /\left(2 t^{2}\right)$, since

$$
\mathscr{F} M \mathscr{U}(-t)\left(i \partial_{t}+\frac{1}{2} \partial_{x}^{2}\right)=\left(i \partial_{t}+\frac{1}{2 t^{2}} \partial_{\xi}^{2}\right) \mathscr{F} M \mathscr{U}(-t),
$$

and

$$
\begin{aligned}
\mathscr{F} M \mathscr{U}(-t) f_{j}(u) & =i \bar{E}(t) \mathscr{D}\left(\frac{1}{t}\right) f_{j}(u) \\
& =i \lambda_{j} \bar{E}(t) \mathscr{D}\left(\frac{1}{t}\right)|u|^{\sigma_{j}-j} u^{j} \\
& =i^{(1-j) / 2} \lambda_{j} \bar{E}^{1-j} t^{\left(1-\sigma_{j}\right) / 2}\left|i \bar{E} \mathscr{D}\left(\frac{1}{t}\right) u\right|^{\sigma_{j}-j}\left(i \bar{E} \mathscr{D}\left(\frac{1}{t}\right) u\right)^{j} \\
& =i^{(1-j) / 2} t^{\left(1-\sigma_{j}\right) / 2} E^{j-1} \lambda_{j}|v|^{\sigma_{j}-j} v^{j}=i^{(1-j) / 2} t^{\left(1-\sigma_{j}\right) / 2} E^{j-1} f_{j}(v) .
\end{aligned}
$$


When we differentiate equation (4), then we encounter an undesirable time growth which appears from $E^{j-1}$, for $j \neq 1$. Therefore we use the method originated by [6] and developed in [9].

We organize our paper as follows. In Section 2 we prove Theorem 1.1. Section 3 is devoted to the proof of Theorem 1.2. In Section 4 we state some estimates for the fractional derivatives from paper [9].

\section{Proof of Theorem 1.1}

In this section we consider the linearized version of (4), which is written as

$$
\left\{\begin{array}{l}
\mathscr{L} v^{(n)}=\sum_{j \neq 0} i^{(1-j) / 2} t^{\left(1-\sigma_{j}\right) / 2} E^{j-1} f_{j}\left(v^{(n-1)}\right), \\
v^{(n)}(1, x)=i^{1 / 2} e^{-i|x|^{2} / 2} u_{0}(x),
\end{array}\right.
$$

where $v(t)=\mathscr{F} M(t) \mathscr{U}(-t) u(t)$ and $\mathscr{L} v^{(0)}=0$, in the Banach space $\mathbf{X}$ with the following norm

$$
\|v\|_{\mathbf{X}}=\sup _{t>1}\left(\left\||\partial|^{\gamma} v\right\|_{\mathbf{L}^{2}}+\left\||\partial|^{\beta} v\right\|_{\mathbf{L}^{2}}+t^{-\Lambda}\left\||\partial|^{\alpha} v\right\|_{\mathbf{L}^{2}}\right),
$$

where $\Lambda=\max \{0, \alpha+5 / 2-\sigma\} / 2,0 \leq \gamma<1 / 2<\beta<\sigma-5 / 2,1 / 2<\alpha<1$, and the fractional derivative $|\partial|^{\beta}=\left(-\partial_{x}^{2}\right)^{\beta / 2}$. By the Sobolev embedding theorem we have

$$
\|v\|_{\mathbf{L}^{\infty}} \leq C\left\||\partial|^{\gamma} v\right\|_{\mathbf{L}^{2}}+C\left\||\partial|^{\beta} v\right\|_{\mathbf{L}^{2}} \leq C\|v\|_{\mathbf{X}} .
$$

We introduce the regularizing operator

$$
\mathscr{K} \phi=\sqrt{t} \int_{\boldsymbol{R}} K(\xi \sqrt{t}, \eta \sqrt{t}) \phi(\xi-\eta) d \eta
$$

with a kernel $K(x, y)=\pi^{-1 / 2}\langle x\rangle e^{-\langle x\rangle^{2} y^{2}}$ to treat the problem in lower order Sobolev spaces and write (5) as

$$
\mathscr{L} v^{(n)}=\sum_{j \neq 0,1} i^{(1-j) / 2} t^{\left(1-\sigma_{j}\right) / 2} E^{j-1} f_{j}\left(\mathscr{K} v^{(n-1)}\right)+R_{1},
$$

where the remainder term

$$
R_{1}=\sum_{j \neq 0,1} i^{(1-j) / 2} t^{\left(1-\sigma_{j}\right) / 2} E^{j-1}\left(f_{j}\left(v^{(n-1)}\right)-f_{j}\left(\mathscr{K} v^{(n-1)}\right)\right)+t^{\left(1-\sigma_{1}\right) / 2} f_{1}\left(v^{(n-1)}\right)
$$

and

$$
v^{(n-1)} \in(\mathbf{X})_{\leq \varepsilon}=\left\{v \in \mathbf{X} ;\|v\|_{\mathbf{X}} \leq \varepsilon\right\}
$$


For simplicity we denote $v=v^{(n)}, w=v^{(n-1)}$. To eliminate the first summand in the right-hand side of (7) we use the identity

$$
\mathscr{L}\left(t^{\mu_{j}} E^{j-1} \phi\right)=\frac{i}{A_{j}} t^{\mu_{j}-1} E^{j-1} \phi+i t^{\mu_{j}-1}(j-1) E^{j-1} \xi \partial_{\xi} \phi+t^{\mu_{j}} E^{j-1} \mathscr{L} \phi,
$$

since $\mathscr{L}\left(t^{\mu_{j}} E^{j-1}\right)=i A_{j}^{-1} t^{\mu_{j}-1} E^{j-1}$, where

$$
A_{j}=\left(\left(\mu_{j}+\frac{j-1}{2}\right)+\frac{i t}{2} j(j-1) \xi^{2}\right)^{-1}
$$

for $j \neq 0,1$. When $(j-1) j \neq 0$, the coefficient $A_{j}$ compensates the time growth, which comes from the derivatives of $E^{j-1}$. So identity (8) is useful for $j \neq 0,1$. However we note that the exponent $E^{j-1}$ is absent in equation (7) for the case of $j=1$. In view of (8), we get from (7)

$$
\begin{aligned}
\mathscr{L}(v & \left.+\sum_{j \neq 0,1} t^{\mu_{j}} E^{j-1} \phi_{j}\right) \\
= & i \sum_{j \neq 0,1} t^{\mu_{j}-1} E^{j-1}\left(\frac{1}{A_{j}} \phi_{j}-i^{(3-j) / 2} t^{\left(3-\sigma_{j}\right) / 2-\mu_{j}} f_{j}(\mathscr{K} w)\right) \\
& +i \sum_{j \neq 0,1}(j-1) t^{\mu_{j}-1} E^{j-1} \xi \partial_{\xi} \phi_{j}+\sum_{j \neq 0,1} t^{\mu_{j}} E^{j-1} \mathscr{L} \phi_{j}+R_{1} .
\end{aligned}
$$

We now choose $\mu_{j}=\left(3-\sigma_{j}\right) / 2$ and

$$
\phi_{j}=i^{(3-j) / 2} A_{j} f_{j}(\mathscr{K} w)=i^{(3-j) / 2} A_{j} \lambda_{j}|\mathscr{K} w|^{\sigma_{j}-j}(\mathscr{K} w)^{j}
$$

to eliminate the first summand in the right-hand side of (9). By the identity

$$
\mathscr{L}(\psi \chi)=\chi \mathscr{L} \psi+t^{-2} \psi_{\xi} \chi_{\xi}+\psi \mathscr{L} \chi
$$

we obtain

$$
\begin{aligned}
\mathscr{L} \phi_{j} & =i^{(3-j) / 2} \mathscr{L}\left(A_{j} f_{j}(\tilde{w})\right) \\
& =i^{(3-j) / 2} f_{j}(\tilde{w}) \mathscr{L} A_{j}+i^{(3-j) / 2} t^{-2}\left(\partial_{\xi} A_{j}\right) \partial_{\xi} f_{j}(\tilde{w})+i^{(3-j) / 2} A_{j} \mathscr{L} f_{j}(\tilde{w})
\end{aligned}
$$

with $\tilde{w}=\mathscr{K} w$. Therefore by virtue of (10), we get from (9)

$$
\begin{aligned}
\mathscr{L}(v & \left.+\sum_{j \neq 0,1} i^{(3-j) / 2} t^{\left(3-\sigma_{j}\right) / 2} E^{j-1} A_{j} f_{j}(\tilde{w})\right) \\
& =\sum_{j \neq 0,1} i^{(3-j) / 2} t^{\left(1-\sigma_{j}\right) / 2} E^{j-1}\left((j-1) i \xi \partial_{\xi} A_{j}+t \mathscr{L} A_{j}\right) f_{j}(\tilde{w})
\end{aligned}
$$




$$
\begin{aligned}
& +\sum_{j \neq 0,1} i^{(3-j) / 2} t^{\left(1-\sigma_{j}\right) / 2} E^{j-1}\left((j-1) i \xi A_{j}+t^{-1} \partial_{\xi} A_{j}\right) \partial_{\xi} f_{j}(\tilde{w}) \\
& +\sum_{j \neq 0,1} i^{(3-j) / 2} t^{\left(3-\sigma_{j}\right) / 2} E^{j-1} A_{j} \mathscr{L} f_{j}(\tilde{w})+R_{1}
\end{aligned}
$$

We denote

$$
\begin{aligned}
& R_{0}=\sum_{j \neq 0,1} i^{(3-j) / 2} t^{\left(3-\sigma_{j}\right) / 2} E^{j-1} A_{j} f_{j}(\tilde{w}), \quad R_{2}=\sum_{j \neq 0,1} t^{\left(1-\sigma_{j}\right) / 2} E^{j-1} a_{j} f_{j}(\tilde{w}), \\
& R_{3}=\sum_{j \neq 0,1} t^{\left(1-\sigma_{j}\right) / 2} E^{j-1} b_{j} \partial_{\xi} f_{j}(\tilde{w}), \quad R_{4}=\sum_{j \neq 0,1} i^{(3-j) / 2} t^{\left(3-\sigma_{j}\right) / 2} E^{j-1} A_{j} \mathscr{L} f_{j}(\tilde{w}),
\end{aligned}
$$

with $a_{j}=i^{(3-j) / 2}\left((j-1) i \xi \partial_{\xi} A_{j}+t \mathscr{L} A_{j}\right)$ and $b_{j}=i^{(3-j) / 2}\left((j-1) i \xi A_{j}+t^{-1} \partial_{\xi} A_{j}\right)$.

Then we obtain our target equation

$$
\mathscr{L}\left(v+R_{0}\right)=\sum_{l=1}^{4} R_{l} .
$$

We apply derivative $|\partial|^{\alpha}$ to equation (12)

$$
\mathscr{L}\left(|\partial|^{\alpha} v+|\partial|^{\alpha} R_{0}\right)=\sum_{l=1}^{3}|\partial|^{\alpha} R_{l} .
$$

Next we estimate the terms $|\partial|^{\alpha} R_{j}$ in equation (13). By the second estimate of Lemma 4.2 it follows that

$$
\begin{aligned}
\left\||\partial|^{\alpha}\left(E^{j-1} \psi\right)\right\|_{\mathbf{L}^{2}} & \leq\left\|E^{j-1}|\partial|^{\alpha} \psi\right\|_{\mathbf{L}^{2}}+\left\|\left[|\partial|^{\alpha}, E^{j-1}\right] \psi\right\|_{\mathbf{L}^{2}} \\
& \leq C\left\||\partial|^{\alpha} \psi\right\|_{\mathbf{L}^{2}}+C t^{\alpha / 2}\left\|B^{\alpha} \psi\right\|_{\mathbf{L}^{2}},
\end{aligned}
$$

where $B=\langle\xi \sqrt{t}\rangle$. Also since $A_{j}=O\left(B^{-2}\right)$ we have

$$
\begin{aligned}
\left\|\left[|\partial|^{\alpha}, A_{j}\right] \psi\right\|_{\mathbf{L}^{2}} & =C\left\|\int_{\boldsymbol{R}}\left(\tilde{A_{j}}-A_{j}\right) \frac{\tilde{\psi} d y}{|y|^{1+\alpha}}\right\|_{\mathbf{L}^{2}} \\
& =C t\left\|\int_{\boldsymbol{R}} \widetilde{A_{j}} A_{j}(2 \xi-y) y \frac{\tilde{\psi} d y}{|y|^{\alpha+1}}\right\|_{\mathbf{L}^{2}} \\
& \leq C t^{1 / 2}\left\|B^{-1} \psi\right\|_{\mathbf{L}^{2}} \int_{\boldsymbol{R}} \frac{d y}{\langle y \sqrt{t}\rangle|y|^{\alpha}} \leq C t^{\alpha / 2}\left\|B^{-1} \psi\right\|_{\mathbf{L}^{2}},
\end{aligned}
$$

where $\tilde{A_{j}}=A_{j}(\cdot-y), \tilde{\psi}=\psi(\cdot-y)$. Hence we find

$$
\left\||\partial|^{\alpha}\left(E^{j-1} A_{j} \psi\right)\right\|_{\mathbf{L}^{2}} \leq C\left\||\partial|^{\alpha} \psi\right\|_{\mathbf{L}^{2}}+C t^{\alpha / 2}\left\|B^{-1} \psi\right\|_{\mathbf{L}^{2}} .
$$


Also by Lemma 4.4 and (6) we obtain

$$
\left\||\partial|^{\alpha} f_{j}(\tilde{w})\right\|_{\mathbf{L}^{2}} \leq C\|\tilde{w}\|_{\mathbf{L}^{\infty}}^{\sigma_{j}-1}\left\||\partial|^{\alpha} \tilde{w}\right\|_{\mathbf{L}^{2}} \leq C \varepsilon^{\sigma_{j}} t^{\Lambda}
$$

and

$$
\left\|B^{-1} f_{j}(\tilde{w})\right\|_{\mathbf{L}^{2}} \leq C\|\tilde{w}\|_{\mathbf{L}^{\infty}}^{\sigma_{j}}\left\|B^{-1}\right\|_{\mathbf{L}^{2}} \leq C \varepsilon^{\sigma_{j}} t^{-1 / 4} .
$$

Hence by (14) we get

$$
\begin{aligned}
\left\||\partial|^{\alpha} R_{0}\right\|_{\mathbf{L}^{2}} & \leq C \sum_{j \neq 0,1} t^{\left(3-\sigma_{j}\right) / 2}\left\||\partial|^{\alpha}\left(E^{j-1} A_{j} f_{j}(\tilde{w})\right)\right\|_{\mathbf{L}^{2}} \\
& \leq C \sum_{j \neq 0,1} t^{\left(3-\sigma_{j}\right) / 2}\left\||\partial|^{\alpha} f_{j}(\tilde{w})\right\|_{\mathbf{L}^{2}}+C \sum_{j \neq 0,1} t^{\left(3-\sigma_{j}+\alpha\right) / 2}\left\|B^{-1} f_{j}(\tilde{w})\right\|_{\mathbf{L}^{2}} \\
& \leq C \sum_{j \neq 0,1} \varepsilon^{\sigma_{j}} t^{\left(3-\sigma_{j}\right) / 2+\Lambda}+C \sum_{j \neq 0,1} \varepsilon^{\sigma_{j}} t^{\left(3-\sigma_{j}+\alpha\right) / 2-1 / 4} \leq C \varepsilon^{\sigma} t^{\Lambda} .
\end{aligned}
$$

In the same manner since $a_{j}=O\left(B^{-2}\right)$ we find

$$
\begin{aligned}
\left\||\partial|^{\alpha} R_{2}\right\|_{\mathbf{L}^{2}} & \leq \sum_{j \neq 0,1} t^{\left(1-\sigma_{j}\right) / 2}\left\||\partial|^{\alpha}\left(E^{j-1} a_{j} f_{j}(\tilde{w})\right)\right\|_{\mathbf{L}^{2}} \\
& \leq C \sum_{j \neq 0,1} \varepsilon^{\sigma_{j}} t^{\left(1-\sigma_{j}\right) / 2+\Lambda}+C \sum_{j \neq 0,1} \varepsilon^{\sigma_{j}} t^{\left(1-\sigma_{j}+\alpha\right) / 2-1 / 4} \\
& \leq C \varepsilon^{\sigma} t^{(1-\sigma) / 2+\Lambda}+C \varepsilon^{\sigma} t^{(\alpha-\sigma+1 / 2) / 2} .
\end{aligned}
$$

Next by the second estimate of Lemma 4.2 we have

$$
\begin{aligned}
\left\||\partial|^{\alpha} R_{1}\right\|_{\mathbf{L}^{2}} \leq & C \sum_{j \neq 0,1} t^{\left(1-\sigma_{j}\right) / 2}\left\||\partial|^{\alpha}\left(f_{j}(w)-f_{j}(\tilde{w})\right)\right\|_{\mathbf{L}^{2}}+C t^{\left(1-\sigma_{1}\right) / 2}\left\||\partial|^{\alpha} f_{1}(w)\right\|_{\mathbf{L}^{2}} \\
& +C \sum_{j \neq 0,1} t^{\left(1-\sigma_{j}+a\right) / 2}\left\|B^{\alpha}\left(f_{j}(w)-f_{j}(\tilde{w})\right)\right\|_{\mathbf{L}^{2}} .
\end{aligned}
$$

Denote $W=w-\tilde{w}$, since $K_{0}=\int_{R} K(\xi \sqrt{t}, y) d y=1$, then by Lemma 4.3 we find

$$
\left\||\partial|^{\alpha} W\right\|_{\mathbf{L}^{2}}+t^{\alpha / 2}\left\|B^{\alpha} W\right\|_{\mathbf{L}^{2}} \leq C\left\||\partial|^{\alpha} w\right\|_{\mathbf{L}^{2}} \leq C \varepsilon t^{\Lambda},
$$

and also

$$
\left\||\partial|^{\alpha} \tilde{w}\right\|_{\mathbf{L}^{2}} \leq\left\||\partial|^{\alpha} W\right\|_{\mathbf{L}^{2}}+\left\||\partial|^{\alpha} w\right\|_{\mathbf{L}^{2}} \leq C \varepsilon t^{\Lambda} .
$$

Then by Lemma 4.4 we obtain

$$
\left\||\partial|^{\alpha}\left(f_{j}(w)-f_{j}(\tilde{w})\right)\right\|_{\mathbf{L}^{2}} \leq C\|w\|_{\mathbf{L}^{\infty}}^{\sigma_{j}-1}\left\||\partial|^{\alpha} w\right\|_{\mathbf{L}^{2}} \leq C \varepsilon^{\sigma} t^{\Lambda}
$$

and 


$$
\begin{aligned}
t^{\alpha / 2}\left\|B^{\alpha}\left(f_{j}(w)-f_{j}(\tilde{w})\right)\right\|_{\mathbf{L}^{2}} & \leq C t^{\alpha / 2}\left(\|w\|_{\mathbf{L}^{\infty}}^{\sigma_{j}-1}+\|\tilde{w}\|_{\mathbf{L}^{\infty}}^{\sigma_{j}-1}\right)\left\|B^{\alpha} W\right\|_{\mathbf{L}^{2}} \\
& \leq C \varepsilon^{\sigma} t^{\Lambda} .
\end{aligned}
$$

Therefore we find

$$
\left\||\partial|^{\alpha} R_{1}\right\|_{\mathbf{L}^{2}} \leq C \varepsilon^{\sigma} t^{(1-\sigma) / 2+\Lambda} .
$$

Next we estimate the term

$$
\begin{aligned}
|\partial|^{\alpha} R_{3} & =\sum_{j \neq 0,1} t^{\left(1-\sigma_{j}\right) / 2}|\partial|^{\alpha}\left(E^{j-1} b_{j} \partial_{\xi} f_{j}(\tilde{w})\right) \\
& =\sum_{j \neq 0,1} t^{\left(1-\sigma_{j}\right) / 2}|\partial|^{\alpha}\left(E^{j-1} b_{j}\left(\partial_{\tilde{w}} f_{j}(\tilde{w}) \partial_{\xi} \tilde{w}+\partial_{\tilde{\tilde{w}}} f_{j}(\tilde{w}) \overline{\partial_{\xi} \tilde{w}}\right)\right) .
\end{aligned}
$$

We have the identity

$$
\begin{aligned}
t^{-1 / 2} B^{-1} \partial_{\xi} \tilde{w} & =\frac{1}{\sqrt{\pi}\langle\xi \sqrt{t}\rangle} \partial_{\xi}\langle\xi \sqrt{t}\rangle \int_{\boldsymbol{R}} e^{-\langle\xi \sqrt{t}\rangle^{2}((\xi-\eta) \sqrt{t})^{2}} w(t, \eta) d \eta \\
& =\mathscr{K}_{1} w
\end{aligned}
$$

where we denote the operator

$$
\mathscr{K}_{1} \phi=\sqrt{t} \int_{\boldsymbol{R}} K_{1}(\xi \sqrt{t}, \eta \sqrt{t}) \phi(\xi-\eta) d \eta,
$$

with kernel

$$
\begin{aligned}
K_{1}(x, y) & =\langle x\rangle^{-1}\left(\partial_{x}+\partial_{y}\right) K(x, y) \\
& =\frac{1}{\sqrt{\pi}}\left(x\langle x\rangle^{-2}\left(1-2\langle x\rangle^{2} y^{2}\right)-2\langle x\rangle^{2} y\right) e^{-\langle x\rangle^{2} y^{2}},
\end{aligned}
$$

which satisfies the conditions of Lemmas 4.1 and 4.3. Also we have

$$
K_{1,0}=\int_{\boldsymbol{R}} K_{1}(x, y) d y=0 .
$$

Note that $b_{j}=i^{(3-j) / 2}\left((j-1) i \xi A_{j}+t^{-1} \partial_{\xi} A_{j}\right)=O\left(t^{-1 / 2} B^{-1}\right)$. Then by Lemma 4.2 we get

$$
\begin{aligned}
\left\||\partial|^{\alpha}\left(E^{j-1} b_{j} \partial_{\tilde{w}} f_{j}(\tilde{w}) \partial_{\tilde{\xi}} \tilde{w}\right)\right\|_{\mathbf{L}^{2}}= & t^{1 / 2}\left\||\partial|^{\alpha}\left(E^{j-1} b_{j} B \partial_{\tilde{w}} f_{j}(\tilde{w}) \mathscr{K}_{1} w\right)\right\|_{\mathbf{L}^{2}} \\
\leq & \left\||\partial|^{\alpha}\left(\partial_{\tilde{w}} f_{j}(\tilde{w})\left(\mathscr{K}_{1}-K_{1,0}\right) w\right)\right\|_{\mathbf{L}^{2}} \\
& +C t^{\alpha / 2}\|\tilde{w}\|_{\mathbf{L}^{\infty}}^{\sigma_{j}-1}\left\|B^{\alpha}\left(\mathscr{K}_{1}-K_{1,0}\right) w\right\|_{\mathbf{L}^{2}} \\
\leq & C \varepsilon^{\sigma} t^{\Lambda}+C \varepsilon^{\sigma} t^{\alpha / 2-1 / 4} .
\end{aligned}
$$


Hence

$$
\begin{aligned}
\left\||\partial|^{\alpha} R_{3}\right\|_{\mathbf{L}^{2}} & \leq \sum_{j \neq 0,1} t^{\left(1-\sigma_{j}\right) / 2}\left\||\partial|^{\alpha}\left(E^{j-1} b_{j}\left(\partial_{\tilde{w}} f_{j}(\tilde{w}) \partial_{\tilde{\xi}} \tilde{w}+\partial_{\tilde{\tilde{w}}} f_{j}(\tilde{w}) \overline{\partial_{\xi} \tilde{w}}\right)\right)\right\|_{\mathbf{L}^{2}} \\
& \leq C \varepsilon^{\sigma} t^{(1-\sigma) / 2+\Lambda}+C \varepsilon^{\sigma} t^{(\alpha-\sigma+1 / 2) / 2} .
\end{aligned}
$$

Finally we estimate the term $|\partial|^{\alpha} R_{4}$. We have

$$
\begin{aligned}
\mathscr{L} f_{j}(\tilde{w})= & \left(\partial_{\tilde{w}} f_{j}(\tilde{w})\right) \mathscr{L} \tilde{w}-\left(\partial_{\tilde{\tilde{w}}} f_{j}(\tilde{w})\right) \overline{\mathscr{L} \tilde{w}} \\
& +\frac{1}{t^{2}}\left(\partial_{\tilde{\tilde{w}}} f_{j}(\tilde{w})\right)\left(\overline{\partial_{\xi}^{2} \tilde{w}}\right) \\
& +\frac{1}{2 t^{2}}\left(\partial_{\tilde{w}}^{2} f_{j}(\tilde{w})\right)\left(\partial_{\xi} \tilde{w}\right)^{2}+\frac{1}{2 t^{2}}\left(\partial_{\tilde{\tilde{w}}}^{2} f_{j}(\tilde{w})\right)\left(\overline{\partial_{\tilde{\xi}} \tilde{w}}\right)^{2} \\
& +\frac{1}{t^{2}}\left(\partial_{\tilde{w}} \partial_{\tilde{\tilde{w}}} f_{j}(\tilde{w})\right)\left|\partial_{\xi} \tilde{w}\right|^{2}
\end{aligned}
$$

We find

$$
\begin{aligned}
\||\partial|^{\alpha} & \left(E^{j-1} A_{j}\left(\partial_{\tilde{w}}^{2} f_{j}(\tilde{w})\right)\left(\partial_{\xi} \tilde{w}\right)^{2}\right) \|_{\mathbf{L}^{2}} \\
\leq & C t\left\||\partial|^{\alpha}\left(E^{j-1} A_{j} B^{2}\left(\partial_{\tilde{w}}^{2} f_{j}(\tilde{w})\right)\left(\mathscr{K}_{1} w\right)^{2}\right)\right\|_{\mathbf{L}^{2}} \\
\leq & C t\left\||\partial|^{\alpha}\left(\left(\partial_{\tilde{w}}^{2} f_{j}(\tilde{w})\right)\left(\left(\mathscr{K}_{1}-K_{1,0}\right) w\right)^{2}\right)\right\|_{\mathbf{L}^{2}} \\
& +C t^{1+\alpha / 2}\|\tilde{w}\|_{\mathbf{L}^{\infty}}^{\sigma_{j}-2}\left\|\mathscr{K}_{1} w\right\|_{\mathbf{L}^{\infty}}\left\|B^{\alpha}\left(\mathscr{K}_{1}-K_{1,0}\right) w\right\|_{\mathbf{L}^{2}} \\
\leq & C \varepsilon^{\sigma} t^{1+\Lambda}+C \varepsilon^{\sigma} t^{\alpha / 2+3 / 4} .
\end{aligned}
$$

Similarly,

$$
\begin{aligned}
\||\partial|^{\alpha}( & \left.E^{j-1} A_{j}\left(\partial_{\tilde{\tilde{w}}}^{2} f_{j}(\tilde{w})\right)\left(\partial_{\xi} \overline{\tilde{w}}\right)^{2}\right) \|_{\mathbf{L}^{2}} \\
& +\left\||\partial|^{\alpha}\left(E^{j-1} A_{j}\left(\partial_{\tilde{w}} \partial_{\tilde{w}} f_{j}(\tilde{w})\right)\left|\partial_{\xi} \tilde{w}\right|^{2}\right)\right\|_{\mathbf{L}^{2}} \\
\leq & C \varepsilon^{\sigma} t^{1+\Lambda}+C \varepsilon^{\sigma} t^{\alpha / 2+3 / 4}
\end{aligned}
$$

In the same manner as in the definition of $\mathscr{K}_{1}$ we write

$$
\begin{aligned}
t^{-1} B^{-2} \partial_{\xi}^{2} \tilde{w} & =\frac{1}{\sqrt{\pi t}\langle\xi \sqrt{t}\rangle^{2}} \partial_{\xi}^{2}\langle\xi \sqrt{t}\rangle \int_{\boldsymbol{R}} e^{-\langle\xi \sqrt{t}\rangle^{2}((\xi-\eta) \sqrt{t})^{2}} w(t, \eta) d \eta \\
& =\mathscr{K}_{2} w
\end{aligned}
$$

where we denote the operator

$$
\mathscr{K}_{2} \phi=\sqrt{t} \int_{\boldsymbol{R}} K_{2}(\xi \sqrt{t}, \eta \sqrt{t}) \phi(\xi-\eta) d \eta
$$


with kernel

$$
K_{2}(x, y)=\langle x\rangle^{-2}\left(\partial_{x}+\partial_{y}\right)^{2} K(x, y),
$$

which satisfies the conditions of Lemmas 4.1 and 4.3. Also we have

$$
K_{2,0}=\int_{\boldsymbol{R}} K_{2}(x, y) d y=0 .
$$

Therefore as above we get

$$
\begin{aligned}
\left\||\partial|^{\alpha}\left(E^{j-1} A_{j}\left(\partial_{\tilde{\tilde{w}}} f_{j}(\tilde{w})\right)\left(\overline{\partial_{\xi}^{2} \tilde{w}}\right)\right)\right\|_{\mathbf{L}^{2}} \\
\leq C t\left\||\partial|^{\alpha}\left(E^{j-1} A_{j} B^{2}\left(\partial_{\tilde{\tilde{w}}} f_{j}(\tilde{w})\right) \overline{\mathscr{K}_{2} w}\right)\right\|_{\mathbf{L}^{2}} \\
\leq C t\left\||\partial|^{\alpha}\left(\left(\partial_{\overline{\tilde{w}}} f_{j}(\tilde{w})\right) \overline{\left(\mathscr{K}_{2}-K_{2,0}\right) w}\right)\right\|_{\mathbf{L}^{2}} \\
\quad+C t^{1+\alpha / 2}\|\tilde{w}\|_{\mathbf{L}^{\infty}}^{\sigma_{j}-1}\left\|B^{\alpha}\left(\mathscr{K}_{2}-K_{2,0}\right) w\right\|_{\mathbf{L}^{2}} \\
\leq C \varepsilon^{\sigma} t^{1+\Lambda}+C \varepsilon^{\sigma} t^{\alpha / 2+3 / 4} .
\end{aligned}
$$

Finally, we consider the term

$$
\sum_{j \neq 0,1} i^{(3-j) / 2} t^{\left(1-\sigma_{j}\right) / 2}|\partial|^{\alpha}\left(E^{j-1} A_{j}\left(\left(\partial_{\tilde{w}} f_{j}(\tilde{w})\right) t \mathscr{L} \tilde{w}-\left(\partial_{\tilde{\tilde{w}}} f_{j}(\tilde{w})\right) \overline{t \mathscr{L} \tilde{w}}\right)\right)
$$

By virtue of equation (5) we have $\mathscr{L} v^{(n-1)}=\sum_{j \neq 0} i^{(1-j) / 2} t^{\left(1-\sigma_{j}\right) / 2} E^{j-1} f_{j}\left(v^{(n-2)}\right)$. Therefore

$$
\begin{aligned}
t \mathscr{L} \tilde{w} & =\mathscr{K}_{3} w+\mathscr{K} t \mathscr{L} w \\
& =\mathscr{K}_{3} w+\sum_{l \neq 0} i^{(1-l) / 2} t^{\left(3-\sigma_{l}\right) / 2} \mathscr{K}\left(E^{l-1} f_{l}\left(v^{(n-2)}\right)\right) \\
& =\mathscr{K}_{3} w+\sum_{l \neq 0} i^{(1-l) / 2} t^{\left(3-\sigma_{l}\right) / 2} E^{l-1} \mathscr{K}_{4, l} f_{l}\left(v^{(n-2)}\right),
\end{aligned}
$$

where

$$
\mathscr{K}_{3} \phi=\sqrt{t} \int_{\boldsymbol{R}} K_{3}(\xi \sqrt{t}, \eta \sqrt{t}) \phi(\xi-\eta) d \eta
$$

with kernel

$$
K_{3}(\xi \sqrt{t}, \eta \sqrt{t})=\frac{1}{\sqrt{\pi}}\left(\sqrt{t} \mathscr{L}\left(\sqrt{t} B e^{-t B^{2} \eta^{2}}\right)+t^{-1} \partial_{\eta} \partial_{\xi}\left(B e^{-t B^{2} \eta^{2}}\right)\right)
$$

and

$$
\mathscr{K}_{4, l} \phi=\sqrt{t} \int_{\boldsymbol{R}} K_{4, l}(\xi \sqrt{t}, \eta \sqrt{t}) \phi(\xi-\eta) d \eta
$$


with kernel

$$
K_{4, l}(x, y)=\frac{\langle x\rangle}{\sqrt{\pi}} e^{-\langle x\rangle^{2} y^{2}+i(l-1)\left(y^{2}-2 x y\right) / 2},
$$

which satisfy the conditions of Lemma 4.1 and Lemma 4.3.

Then as above we get

$$
\begin{aligned}
\||\partial|^{\alpha}( & \left.E^{j-1} A_{j}\left(\partial_{\tilde{w}} f_{j}(\tilde{w})\right) t \mathscr{L} \tilde{w}\right) \|_{\mathbf{L}^{2}} \\
\leq & \left\||\partial|^{\alpha}\left(E^{j-1} A_{j}\left(\partial_{\tilde{w}} f_{j}(\tilde{w})\right) \mathscr{K}_{3} w\right)\right\|_{\mathbf{L}^{2}} \\
& +\sum_{l \neq 0} t^{\left(3-\sigma_{l}\right) / 2}\left\||\partial|^{\alpha}\left(E^{j+l-2} A_{j}\left(\partial_{\tilde{w}} f_{j}(\tilde{w})\right) \mathscr{K}_{4, l} f_{l}\left(v^{(n-2)}\right)\right)\right\|_{\mathbf{L}^{2}} \\
\leq & C\left\||\partial|^{\alpha}\left(\partial_{\tilde{w}} f_{j}(\tilde{w}) \mathscr{K}_{3} w\right)\right\|_{\mathbf{L}^{2}}+C t^{\alpha / 2}\left\|B^{-1} \partial_{\tilde{w}} f_{j}(\tilde{w}) \mathscr{K}_{3} w\right\|_{\mathbf{L}^{2}} \\
& +\sum_{l \neq 0}\left\||\partial|^{\alpha}\left(\left(\partial_{\tilde{w}} f_{j}(\tilde{w})\right) \mathscr{K}_{4, l} f_{l}\left(v^{(n-2)}\right)\right)\right\|_{\mathbf{L}^{2}} \\
& +C \sum_{l \neq 0} t^{\alpha / 2}\left\|B^{-1}\left(\partial_{\tilde{w}} f_{j}(\tilde{w})\right) \mathscr{K}_{4, l} f_{l}\left(v^{(n-2)}\right)\right\|_{\mathbf{L}^{2}} \leq C \varepsilon^{\sigma} t^{\Lambda}+C \varepsilon^{\sigma} t^{\alpha / 2-1 / 4} .
\end{aligned}
$$

Similarly,

$$
\left\||\partial|^{\alpha}\left(E^{j-1} A_{j}\left(\partial_{\tilde{\tilde{w}}} f_{j}(\tilde{w})\right) \overline{t \mathscr{L} \tilde{w}}\right)\right\|_{\mathbf{L}^{2}} \leq C \varepsilon^{\sigma} t^{\Lambda}+C \varepsilon^{\sigma} t^{\alpha / 2-1 / 4} .
$$

Collecting estimates (19)-(23) we obtain

$$
\left\||\partial|^{\alpha} R_{4}\right\|_{\mathbf{L}^{2}} \leq C \varepsilon^{\sigma} t^{(1-\sigma) / 2+\Lambda}+C \varepsilon^{\sigma} t^{(\alpha-\sigma+1 / 2) / 2} .
$$

Now substituting estimates (16), (17), (18) and (24) into (13) we find

$$
\frac{d}{d t}\left\||\partial|^{\alpha}\left(v+R_{0}\right)\right\|_{\mathbf{L}^{2}} \leq C \varepsilon^{\sigma} t^{(1-\sigma) / 2+\Lambda}+C \varepsilon^{\sigma} t^{(\alpha-\sigma+1 / 2) / 2} .
$$

The integration with respect to time yields

$$
\left\||\partial|^{\alpha}\left(v+R_{0}\right)\right\|_{\mathbf{L}^{2}} \leq C \varepsilon+C \varepsilon^{\sigma} t^{\Lambda}
$$

from which in view of (15) it follows that

$$
\left\||\partial|^{\alpha} v^{(n)}\right\|_{\mathbf{L}^{2}} \leq C \varepsilon+C \varepsilon^{\sigma} t^{\Lambda}
$$

In the same way as above, by (12) we find the estimates

$$
\left\||\partial|^{\gamma} v^{(n)}\right\|_{\mathbf{L}^{2}}+\left\||\partial|^{\beta} v^{(n)}\right\|_{\mathbf{L}^{2}} \leq C \varepsilon+C \varepsilon^{\sigma}
$$

and

$$
\left\||\partial|^{\alpha}\left(v^{(n)}(t)-v^{(n-1)}(t)\right)\right\|_{\mathbf{L}^{2}} \leq C \varepsilon^{\sigma} t^{\Lambda},
$$


which mean that $\left\{v^{(n)}\right\}$ is a Cauchy sequence in $\mathbf{X}$. Therefore we have a unique solution $v$ satisfying the estimates

$$
\begin{aligned}
\left\||\partial|^{\alpha} v\right\|_{\mathbf{L}^{2}} & \leq C \varepsilon+C \varepsilon^{\sigma} t^{\Lambda}, \\
\left\||\partial|^{\gamma} v\right\|_{\mathbf{L}^{2}}+\left\||\partial|^{\beta} v\right\|_{\mathbf{L}^{2}} & \leq C \varepsilon+C \varepsilon^{\sigma}
\end{aligned}
$$

and the integral equation associated with equation

$$
\mathscr{L}\left(v+R_{0}\right)=\sum_{l=1}^{4} R_{l},
$$

where

$$
\begin{aligned}
& R_{0}=\sum_{j \neq 0,1} i^{(3-j) / 2} t^{\left(3-\sigma_{j}\right) / 2} E^{j-1} A_{j} f_{j}(\mathscr{K} v), \quad R_{2}=\sum_{j \neq 0,1} t^{\left(1-\sigma_{j}\right) / 2} E^{j-1} a_{j} f_{j}(\mathscr{K} v), \\
& R_{1}=\sum_{j \neq 0,1} i^{(1-j) / 2} t^{\left(1-\sigma_{j}\right) / 2} E^{j-1}\left(f_{j}(v)-f_{j}(\mathscr{K} v)\right)+t^{\left(1-\sigma_{1}\right) / 2} f_{1}(v), \\
& R_{3}=\sum_{j \neq 0,1} t^{\left(1-\sigma_{j}\right) / 2} E^{j-1} b_{j} \partial_{\xi} f_{j}(\mathscr{K} v), \quad R_{4}=\sum_{j \neq 0,1} i^{(3-j) / 2} t^{\left(3-\sigma_{j}\right) / 2} E^{j-1} A_{j} \mathscr{L} f_{j}(\mathscr{K} v),
\end{aligned}
$$

with $a_{j}=i^{(3-j) / 2}\left((j-1) i \xi \partial_{\xi} A_{j}+t \mathscr{L} A_{j}\right)$ and $b_{j}=i^{(3-j) / 2}\left((j-1) i \xi A_{j}+t^{-1} \partial_{\xi} A_{j}\right)$. By (27) it is clear that $\|v\|_{\mathbf{X}}<\infty$. From (28) and the above estimates for $R_{j}$, it follows that

$$
\left\|v(t)-v(s)+R_{0}(t)-R_{0}(s)\right\|_{\dot{\mathbf{H}}^{\gamma} \cap \dot{\mathbf{H}}^{\beta}} \leq C \varepsilon^{\sigma} s^{(\beta+5 / 2-\sigma) / 2}
$$

for $t>s>1$ and $0 \leq \gamma<1 / 2<\beta<p-3 / 2$. Also by (15) we have

$$
\left\|R_{0}(s)\right\|_{\dot{\mathbf{H}}^{\gamma} \cap \dot{\mathbf{H}}^{\beta}} \leq C \varepsilon^{\sigma} S^{(\beta+5 / 2-\sigma) / 2} .
$$

Therefore

$$
\begin{aligned}
\|M(t) \mathscr{U}(-t) u(t)-M(s) \mathscr{U}(-s) u(s)\|_{\dot{\mathbf{H}}^{0, \gamma} \cap \dot{\mathbf{H}}^{0, \beta}} \\
\quad=\|\mathscr{F} M(t) \mathscr{U}(-t) u(t)-\mathscr{F} M(s) \mathscr{U}(-s) u(s)\|_{\dot{\mathbf{H}}^{\gamma} \cap \dot{\mathbf{H}}^{\beta}} \\
\quad=\|v(t)-v(s)\|_{\dot{\mathbf{H}}^{\gamma} \cap \dot{\mathbf{H}}^{\beta}} \leq C \varepsilon s^{(\beta+5 / 2-\sigma) / 2} \rightarrow 0
\end{aligned}
$$

for $t>s \rightarrow \infty$. Then there exists a unique scattering state $u_{+} \in \dot{\mathbf{H}}^{0, \gamma} \cap \dot{\mathbf{H}}^{0, \beta}$ such that

$$
\begin{aligned}
& \left\|\mathscr{U}(-t) u(t)-M(-t) u_{+}\right\|_{\dot{\mathbf{H}}^{0, \gamma} \cap \dot{\mathbf{H}}^{0, \beta}} \\
& \quad=\left\|M(t) \mathscr{U}(-t) u(t)-u_{+}\right\|_{\dot{\mathbf{H}}^{0, \gamma} \cap \dot{\mathbf{H}}^{0, \beta}} \leq C \varepsilon t^{(\beta+5 / 2-\sigma) / 2}
\end{aligned}
$$


Hence

$$
\begin{aligned}
& \| \mathscr{U}(-t) u(t)-u_{+} \|_{\dot{\mathbf{H}}^{0, \gamma} \cap \dot{\mathbf{H}}^{0, \beta}} \\
& \quad \leq\left\|\mathscr{U}(-t) u(t)-M(-t) u_{+}\right\|_{\dot{\mathbf{H}}^{0, \gamma} \cap \dot{\mathbf{H}}^{0, \beta}}+\left\|M(-t) u_{+}-u_{+}\right\|_{\dot{\mathbf{H}}^{0, \gamma} \cap \dot{\mathbf{H}}^{0, \beta}} \\
& \quad \leq C \varepsilon t^{(\beta+5 / 2-\sigma) / 2}+\left\|M(-t) u_{+}-u_{+}\right\|_{\dot{\mathbf{H}}^{0, \gamma} \cap \dot{\mathbf{H}}^{0, \beta}} \rightarrow 0
\end{aligned}
$$

as $t \rightarrow \infty$. Theorem 1.1 is proved.

\section{Proof of Theorem 1.2}

Since the norm $\left\||\partial|^{\alpha} \phi\right\|_{\mathbf{L}^{2}}$ of the homogeneous Sobolev space $\dot{\mathbf{H}}^{\alpha}$ is equivalent to that of the homogeneous Besov space $\dot{\mathbf{B}}_{2,2}^{\alpha}$ (see [1]), we have

$$
\begin{aligned}
\left\|e^{i t x^{2}} \phi\right\|_{\dot{\mathbf{H}}^{\alpha}} & \leq C\left\|e^{i t x^{2}} \phi\right\|_{\dot{\mathbf{B}}_{2,2}^{\alpha}} \\
& =C\left(\int_{0}^{\infty} x^{-1-2 \alpha} \sup _{|y| \leq x}\left\|e^{i t(z+y)^{2}} \phi(z+y)-e^{i t z^{2}} \phi(z)\right\|_{\mathbf{L}_{z}^{2}}^{2} d x\right)^{1 / 2} .
\end{aligned}
$$

By a simple computation we find

$$
\begin{aligned}
& \left|e^{i t(z+y)^{2}} \phi(z+y)-e^{i t z^{2}} \phi(z)\right| \\
& \quad \leq\left|\left(e^{i t\left((z+y)^{2}-z^{2}\right)}-1\right) \phi(z+y)\right|+|\phi(z+y)-\phi(z)| \\
& \quad \leq C|t(y(2 z+y))|^{\delta}|\phi(z+y)|+|\phi(z+y)-\phi(z)|
\end{aligned}
$$

for $0 \leq \delta \leq 1$. Substituting the last inequality into the above we get

$$
\begin{aligned}
\left\|e^{i t x^{2}} \phi\right\|_{\mathbf{H}^{\alpha}} \leq & C t^{\delta}\left(\int_{0}^{1} x^{-1-2 \alpha} \sup _{|y| \leq x} \int|y|^{2 \delta}|2 z+y|^{2 \delta}|\phi(z+y)|^{2} d z d x\right)^{1 / 2} \\
& +C\left(\int_{1}^{\infty} x^{-1-2 \alpha} \sup _{|y| \leq x} \int|\phi(z+y)|^{2} d z d x\right)^{1 / 2}+C\|\phi\|_{\dot{\mathbf{H}}^{\alpha}} \\
\leq & C t^{\delta}\left(\int_{0}^{1} x^{-1-2 \alpha+2 \delta} \sup _{|y| \leq x} \int\left(|z+y|^{2 \delta}+1\right)|\phi(z+y)|^{2} d z d x\right)^{1 / 2} \\
& +C\|\phi\|_{\dot{\mathbf{H}}^{\alpha}}+C\|\phi\|_{\mathbf{L}^{2}} \\
\leq & C\|\phi\|_{\mathbf{H}^{\alpha}}+C t^{\delta}\|\phi\|_{\mathbf{H}^{0, \delta}}
\end{aligned}
$$


where $\alpha<\delta$. Hence

$$
\begin{aligned}
\|\mathscr{U}(-t) u\|_{\mathbf{H}^{0, \alpha}} & \leq\left\|e^{i t \xi^{2} / 2} \hat{u}\right\|_{\dot{\mathbf{H}}^{\alpha}} \\
& \leq C\left(\|\hat{u}\|_{\mathbf{H}^{\alpha}}+t^{\delta}\|\hat{u}\|_{\mathbf{H}^{0, \delta}} \leq C\left(\|u\|_{\mathbf{H}^{0, \alpha}}+t^{\delta}\|u\|_{\mathbf{H}^{\delta}}\right) .\right.
\end{aligned}
$$

As in the proof of Theorem 1.1, we easily see that if the initial data $u_{0}=$ $u(1, x) \in \mathbf{H}^{\delta} \cap \mathbf{H}^{0, \alpha}$ with $1 / 2<\alpha<\delta<1$, then there exists $\varepsilon_{0}>0$ such that (1) has a unique global solution

$$
\mathscr{U}(-t) u \in \mathbf{C}\left([1, \infty) ; \mathbf{H}^{\delta} \cap \mathbf{H}^{0, \alpha}\right) .
$$

Indeed the sharp time decay estimate $\|u(t)\|_{\mathbf{L}^{\infty}} \leq C t^{-1 / 2}$ obtained in Theorem 1.1 and the integral equation

$$
u(t)=\mathscr{U}(t) u(1)-i \int_{1}^{t} \mathscr{U}(t-s) f(u) d s
$$

associated with (1) yield

$$
u \in \mathbf{C}\left([1, \infty) ; \mathbf{H}^{\delta}\right) .
$$

Let us consider equation (30) for $t \leq 1$. By (29), we see that existence time can be prolonged to $t=0$, and

$$
\mathscr{U}(-t) u \in \mathbf{C}\left([0, \infty) ; \mathbf{H}^{\delta} \cap \mathbf{H}^{0, \alpha}\right) .
$$

This completes the proof of Theorem 1.2.

\section{Appendix}

We first state three Lemmas which were shown in [9]. Define the operator

$$
\mathscr{K} \phi=\sqrt{t} \int_{\boldsymbol{R}} K(\xi \sqrt{t}, \eta \sqrt{t}) \phi(\xi-\eta) d \eta
$$

for $t \geq 1$, where $K(x, y)$ is a given kernel. Denote $B=\langle\xi \sqrt{t}\rangle$.

Lemma 4.1. Let the kernel $K$ satisfy the estimates

$$
|K(x, y)| \leq\langle x\rangle K_{1}(\langle x\rangle y)
$$

for $x, y \in \boldsymbol{R}$, and

$$
|K(x, y)| \leq K_{2}(y),
$$

for $|y| \geq|x| / 2$, where $K_{1}, K_{2} \in \mathbf{L}^{1}$. Then for any $\phi \in \mathbf{L}^{2}$ the estimate is true

$$
\|\mathscr{K} \phi\|_{\mathbf{L}^{2}} \leq C\|\phi\|_{\mathbf{L}^{2}} \text {. }
$$


Moreover if $K_{1} \in \mathbf{L}^{1, \beta}$, with $\beta \geq 0$, then

$$
\left\|B^{-\beta} \mathscr{K} \phi\right\|_{\mathbf{L}^{\infty}} \leq C\left\|B^{-\beta} \phi\right\|_{\mathbf{L}^{\infty}}
$$

provided that the right-hand side is finite.

We denote the fractional derivative for $\alpha \in(0,1)$ (see [16])

$$
|\partial|^{\alpha} \phi=\mathscr{F}^{-1}\left(|\xi|^{\alpha} \hat{\phi}(\xi)\right)=C \int_{\boldsymbol{R}}(\tilde{\phi}-\phi) \frac{d y}{|y|^{1+\alpha}},
$$

where we denote

$$
\tilde{\phi}=\phi(\cdot-y), \quad C=-\frac{\alpha}{2 \Gamma(1-\alpha) \cos (\pi \alpha / 2)},
$$

$\Gamma$ is the Euler gamma function. We can write the Leibnitz rule $|\partial|^{\alpha}(\phi \psi)=$ $\phi|\partial|^{\alpha} \psi+\left[|\partial|^{\alpha}, \phi\right] \psi$ with the commutator

$$
\left[|\partial|^{\alpha}, \phi\right] \psi=C \int_{\boldsymbol{R}}(\tilde{\phi}-\phi) \tilde{\psi} \frac{d y}{|y|^{1+\alpha}} .
$$

In the next lemma we find some estimates for the commutator $\left[|\partial|^{\alpha}, \phi\right] \psi$ for $\alpha \in(0,1)$.

Lemma 4.2. The inequalities are valid

$$
\left\|\left[|\partial|^{\alpha}, \phi\right] \psi\right\|_{\mathbf{L}^{2}} \leq C t^{\alpha / 2}\left(\left\|B^{v} \phi\right\|_{\mathbf{L}^{\infty}}+t^{-1 / 2}\left\|B^{v} \partial \phi\right\|_{\mathbf{L}^{\infty}}\right)\left\|B^{-v} \psi\right\|_{\mathbf{L}^{2}},
$$

where $0 \leq v<\alpha<1$, and

$$
\left\|\left[|\partial|^{\alpha}, \phi\right] \psi\right\|_{\mathbf{L}^{2}} \leq C t^{\alpha / 2}\left(\|\phi\|_{\mathbf{L}^{\infty}}+t^{-1 / 2}\left\|B^{-1} \partial \phi\right\|_{\mathbf{L}^{\infty}}\right)\left\|B^{\alpha} \psi\right\|_{\mathbf{L}^{2}}
$$

provided that the right-hand sides are finite.

Denote

$$
K_{0}(\xi \sqrt{t})=\sqrt{t} \int_{\boldsymbol{R}} K(\xi \sqrt{t}, \eta \sqrt{t}) d \eta=\int_{\boldsymbol{R}} K(\xi \sqrt{t}, \zeta) d \zeta .
$$

In the next lemma we estimate the difference

$$
\mathscr{K} \phi-K_{0} \phi=\sqrt{t} \int_{\boldsymbol{R}} K(\xi \sqrt{t}, \eta \sqrt{t})(\phi(\xi-\eta)-\phi(\xi)) d y .
$$

A typical choice of a kernel is $K(x, y)=\pi^{-1 / 2}\langle x\rangle e^{-\langle x\rangle^{2} y^{2}}$, when applying Lemma 4.3.

Lemma 4.3. Let the kernel $K \in \mathbf{C}^{1}\left(\boldsymbol{R}^{2}\right)$ satisfy the estimates

$$
|K(x, y)| \leq C\langle x\rangle\langle\langle x\rangle y\rangle^{-3}
$$


and

$$
\left|\partial_{x} K(x, y)\right| \leq C\langle y\rangle^{-3}
$$

for all $x, y \in \boldsymbol{R}$. Then the estimate is true

$$
\left\||\partial|^{\alpha}\left(\mathscr{K} \phi-K_{0} \phi\right)\right\|_{\mathbf{L}^{2}}+t^{\alpha / 2}\left\|B^{\alpha}\left(\mathscr{K} \phi-K_{0} \phi\right)\right\|_{\mathbf{L}^{2}} \leq C\left\||\partial|^{\alpha} \phi\right\|_{\mathbf{L}^{2}}
$$

for all $t \geq 1$.

We need the following lemma to consider the fractional order nonlinearities.

Lemma 4.4. Let $0<\alpha<1$. Let $f$ satisfy

$$
|f(\phi)-f(\psi)| \leq C(|\phi|+|\psi|)^{q-1}|\phi-\psi|
$$

where $q>1$. Then the inequalities

$$
\left\||\partial|^{\alpha} f(\phi)\right\|_{\mathbf{L}^{2}} \leq C\|\phi\|_{\mathbf{L}^{\infty}}^{q-1}\left\||\partial|^{\alpha} \phi\right\|_{\mathbf{L}^{2}}
$$

and

$$
\left\||\partial|^{\alpha}(f(\phi) \psi)\right\|_{\mathbf{L}^{2}} \leq C\|\phi\|_{\mathbf{L}^{\infty}}^{q-1}\left(\|\phi\|_{\mathbf{L}^{\infty}}\left\||\partial|^{\alpha} \psi\right\|_{\mathbf{L}^{2}}+\|\psi\|_{\mathbf{L}^{\infty}}\left\||\partial|^{\alpha} \phi\right\|_{\mathbf{L}^{2}}\right)
$$

are valid, provided that the right-hand sides are finite.

Proof. We have

$$
\begin{aligned}
\|f(\phi)\|_{\dot{\mathbf{H}}^{\alpha}} & \leq C\|f(\phi)\|_{\dot{\mathbf{B}}_{2,2}^{\alpha}} \\
& =C\left(\int_{0}^{\infty} x^{-1-2 \alpha} \sup _{|y| \leq x}\|f(\phi(\cdot+y))-f(\phi(\cdot))\|_{\mathbf{L}^{2}}^{2} d x\right)^{1 / 2} \\
& \leq C\|\phi\|_{\mathbf{L}^{\infty}}^{q-1}\left(\int_{0}^{\infty} x^{-1-2 \alpha} \sup _{|y| \leq x}\|\phi(\cdot+y)-\phi(\cdot)\|_{\mathbf{L}^{2}}^{2} d x\right)^{1 / 2} \\
& \leq C\|\phi\|_{\mathbf{L}^{\infty}}^{q-1}\|\phi\|_{\dot{\mathbf{B}}_{2,2}^{\alpha}}=C\|\phi\|_{\mathbf{L}^{\infty}}^{q-1}\|\phi\|_{\mathbf{H}^{\alpha}} .
\end{aligned}
$$

In the same manner we find

$$
\begin{aligned}
\|f(\phi) \psi\|_{\dot{\mathbf{H}}^{\alpha}} & \leq C\|f(\phi) \psi\|_{\dot{\mathbf{B}}_{2,2}^{\alpha}} \\
& =C\left(\int_{0}^{\infty} x^{-1-2 \alpha} \sup _{|y| \leq x}\|f(\phi(\cdot+y)) \psi(\cdot+y)-f(\phi(\cdot)) \psi(\cdot)\|_{\mathbf{L}^{2}}^{2} d x\right)^{1 / 2} \\
& \leq C\|\phi\|_{\mathbf{L}^{\infty}}^{q-1}\|\psi\|_{\mathbf{L}^{\infty}}\left(\int_{0}^{\infty} x^{-1-2 \alpha} \sup _{|y| \leq x}\|\phi(\cdot+y)-\phi(\cdot)\|_{\mathbf{L}^{2}}^{2} d x\right)^{1 / 2}
\end{aligned}
$$




$$
\begin{aligned}
& +C\|\phi\|_{\mathbf{L}^{\infty}}^{q}\left(\int_{0}^{\infty} x^{-1-2 \alpha} \sup _{|y| \leq x}\|\psi(\cdot+y)-\psi(\cdot)\|_{\mathbf{L}^{2}}^{2} d x\right)^{1 / 2} \\
\leq & C\|\phi\|_{\mathbf{L}^{\infty}}^{q-1}\left(\|\psi\|_{\mathbf{L}^{\infty}}\|\phi\|_{\dot{\mathbf{B}}_{2,2}^{\alpha}}+C\|\phi\|_{\mathbf{L}^{\infty}}\|\psi\|_{\dot{\mathbf{B}}_{2,2}^{\alpha}}\right) \\
= & C\|\phi\|_{\mathbf{L}^{\infty}}^{q-1}\left(\|\psi\|_{\mathbf{L}^{\infty}}\|\phi\|_{\dot{\mathbf{H}}^{\alpha}}+\|\phi\|_{\mathbf{L}^{\infty}}\|\psi\|_{\dot{\mathbf{H}}^{\alpha}}\right) \\
\leq & C\|\phi\|_{\mathbf{L}^{\infty}}^{q-1}\left(\|\phi\|_{\mathbf{L}^{\infty}}\left\||\partial|^{\alpha} \psi\right\|_{\mathbf{L}^{2}}+\|\psi\|_{\mathbf{L}^{\infty}}\left\||\partial|^{\alpha} \phi\right\|_{\mathbf{L}^{2}}\right) .
\end{aligned}
$$

Lemma 4.4 is proved.

Acknowledgement. The work of N.H. is supported by JSPS KAKENHI Grant Numbers 24654034, 25220702. The work of P.I.N. is partially supported by CONACYT and PAPIT project IN100113. One of the authors (N.H.) would like to thank professor Kenji Nakanishi for his useful comments on this paper.

\section{References}

[1] Bergh, J. and Löfström, J., Interpolation spaces. An introduction, Grundlehren der Mathematischen Wissenschaften, No. 223, Springer-Verlag, Berlin-New York, 1976.

[2] Cazenave, Th., Semilinear Schrödinger equations, Courant Lecture Notes in Mathematics, 10, New York University, Courant Institute of Mathematical Sciences, New York; American Mathematical Society, Providence, RI, 2003.

[3] Cohn, S., Resonance and long time existence for the quadratic semilinear Schrödinger equation, Comm. Pure Appl. Math., 45 (1992), 973-1001.

[4] Colin, M. and Colin, T., On a quasilinear Zakharov system describing laser-plasma interactions, Differental Integral Equations, 17 (2004), 297-330.

[5] Georgiev, V. and Lucente, S., Decay for nonlinear Klein-Gordon equations, NoDEA Nonlinear Differential Equations Appl., 11 (2004), 529-555.

[6] Hayashi, N. and Naumkin, P. I., Large time behavior of solutions for derivative cubic nonlinear Schrödinger equations without a self-conjugate property, Funkcial. Ekvac., 42 (1999), 311-324

[7] Hayashi, N. and Naumkin, P. I., Domain and range of the modified wave operator for Schrödinger equations with a critical nonlinearity, Comm. Math. Phys., 267 (2006), 477-492.

[8] Hayashi, N. and Naumkin, P. I., Scattering Operator for Nonlinear Klein-Gordon Equations, Commun. Contemp. Math., 11 (2009), 771-781.

[9] Hayashi, N. and Naumkin, P. I., Global existence for the cubic nonlinear Schrödinger equation in lower order Sobolev spaces, Differential Integral Equations, 24 (2011), 801-828.

[10] Hayashi, N., Li, C. and Naumkin, P. I., On a system of nonlinear Schrödinger equations in 2d, Differental Integral Equations, 24 (2011), 417-434.

[11] Hayashi, N., Li, C. and Ozawa, T., Small data scattering for a system of nonlinear Schrödinger equations, Differ. Equ. Appl., 3 (2011), 415-426.

[12] Hayashi, N. and Ozawa, T., Scattering theory in the weighted $\mathbf{L}^{2}\left(\mathbf{R}^{n}\right)$ spaces for some Schrödinger equations, Ann. Inst. H. Poincaré Phys. Théor., 48 (1988), 17-37. 
[13] Klainerman, S., Global existence of small amplitude solutions to nonlinear Klein-Gordon equations in four space-time dimensions, Comm. Pure Appl. Math., 38 (1985), 631-641.

[14] Kosecki, R., The unit condition and global existence for a class of nonlinear Klein-Gordon equations, J. Differential Equations, 100 (1992), 257-268.

[15] Shatah, J., Normal forms and quadratic nonlinear Klein-Gordon equations, Comm. Pure Appl. Math., 38 (1985), 685-696.

[16] Stein, E. M., Singular Integrals and Differentiability Properties of Functions, Princeton Mathematical Series, No. 30, Princeton University Press, Princeton, NJ, 1970.

[17] Strauss, W. A., Nonlinear scattering theory, Scattering Theory in Mathematical Physics, Reidel, Dordrect, 1979, pp. 53-79.

[18] Strauss, W. A., Nonlinear scattering theory at low energy, J. Funct. Anal., 41 (1981), 110-133.

[19] Sunagawa, H., On global small amplitude solutions to systems of cubic nonlinear KleinGordon equations with different mass in one space dimension, J. Differential Equations, 192 (2003), 308-325.

\author{
nuna adreso: \\ Nakao Hayashi \\ Department of Mathematics \\ Garduate School of Science \\ Osaka University \\ Japan \\ E-mail: nhayashi@math.sci.osaka-u.ac.jp \\ Pavel I. Naumkin \\ Instituto de Matemáticas \\ UNAM Campus Morelia, AP 61-3 (Xangari) \\ Morelia CP 58089, Michoacán \\ Mexico \\ E-mail: pavelni@matmor.unam.mx
}

(Ricevita la 28-an de novembro, 2013)

(Reviziita la 26-an de aŭgusto, 2014) 